\title{
Correction: The effects of melatonin supplementation on blood pressure in patients with metabolic disorders: a systematic review and meta-analysis of randomized controlled trials
}

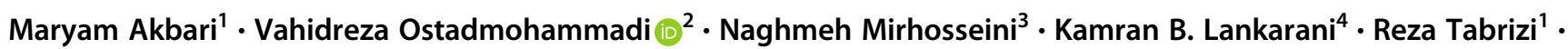 \\ Zahra Keshtkaran $^{5} \cdot$ Russel J. Reiter ${ }^{6} \cdot$ Zatollah Asemi $^{2}$
}

Published online: 23 October 2019

(c) The Author(s), under exclusive licence to Springer Nature Limited 2019

\section{Correction to: Journal of Human Hypertension https://doi.org/10.1038/s41371-019-0166-2}

In the original Review Article, it was discovered that there were methodological issues that invalidated the reported results of the meta-analyses performed in the study, as well as a number of references and citations not being entered correctly. In addition, concerns were raised regarding the present paper and the results and conclusion, namely in regards to the effect sizes used in the present study. In light of these errors the authors re-assessed and re-ran the whole analyses and the below corrections now apply:

(1) Unfortunately, a few references were inserted wrongly and the mistakes occurred due to an old version of Endnote being used. The correct references are as follows:

Pakravan H, Ahmadian M, Fani A, Aghaee D, Brumanad S, Pakzad B. The effects of melatonin in patients with nonalcoholic fatty liver disease: a randomized controlled trial. Adv Biomed Res. 2017;6:40.

Grossman et al. [20] and Agahi et al. [21] are correct in Table 1.

(2) Regarding the method of analysis, the authors reviewed different approaches in conducting a meta-analysis and although their method (of using Borenstein et al. 2011 and Follmann et al. 1992) was not wrong, they concluded that the Cohen statistic method is more accurate. After applying the Cohen statistic method, the calculated effect sizes have been changed; however, the direction of the effect sizes or the level of significant are similar to our original analyses; therefore, the conclusion did not change. The pooled findings indicated a significant reduction in systolic (SBP) $(\mathrm{SMD}=-0.42 ; 95 \%$ CI, $\left.-0.59,-0.25 ; P<0.001 ; I^{2}: 0.0\right)$ and diastolic blood pressure (DBP) (SMD $=-0.50 ; 95 \% \mathrm{CI},-0.72,-0.27 ; P<$ $\left.0.001 ; I^{2}: 37.1\right)$ following melatonin supplementation in individuals with metabolic disorders.
Zatollah Asemi

asemi_r@yahoo.com

1 Health Policy Research Center, Institute of Health, Student Research Committee, Shiraz University of Medical Sciences, Shiraz, Iran

2 Research Center for Biochemistry and Nutrition in Metabolic Diseases, Kashan University of Medical Sciences, Kashan, Iran

3 School of Public Health, University of Saskatchewan, Saskatoon, SK, Canada
4 Health Policy Research Center, Institute of Health, Shiraz University of Medical Sciences, Shiraz, Iran

5 Community Health Nursing Department, Community Based Psychiatric Care Research Center, Shiraz University of Medical Sciences, Shiraz, Iran

6 Department of Cellular and Structural Biology, University of Texas Health Science Center, San Antonio, TX, USA 